\title{
Anchor truncation scheme optimization and its application
}

\author{
Fengshan $\mathrm{Han}^{1, \mathrm{a}}$, Xinli $\mathrm{Wu}^{2 \mathrm{~b}}$ \\ ${ }^{1}$ Research Center for Numerical Tests on Material Failure, Dalian University, Dalian, 116622, China \\ ${ }^{2}$ College of Engineering, the Pennsylvania State University, Pennsylvania, USA \\ ahanfs@sohu.com bxinli@psu.edu \\ Corresponding author: hanfs@sohu.com
}

\section{Keywords: Anchor or Bolt; Truncation Scheme; Optimization; Application}

\begin{abstract}
Coal mine in China is mainly underground mining, it need a large number of underground roadway engineering, to keep roadway and surrounding rock stability of coal mine construction and production safety is of great significance. Bolt or anchor support in coal mines has become a main supporting way in coal mine roadway, So in coal mine underground roadway engineering, a lot of different length of the bolt or anchor are required. For a certain length of steel bar, when a large number of different length of anchor or bolt are used, how to choose Truncation scheme or cutting scheme, how to make the consume the least amount of steel ? to solve this problem, In this paper, authors set up the linear programming optimization mathematical model, using System engineering optimization ideas and methods, it can be easily used for concrete engineering practice. Thus, using system engineering optimization ideas and methods to solve practical engineering problems is very meaningful.
\end{abstract}

\section{Introduction}

Bolt support as an effective way of roadway support, can significantly improve the stability of roadway surrounding rock structure, low economic cost, ease of installation and improve the advantages of safety in production, have been widely used in mine, building, water and electricity and defense engineering fields. Bolt support technology has become indispensable support method in mining and geotechnical engineering.

Coal mine in China is mainly underground mining, it need a large number of underground roadway engineering, according to incomplete statistics, the total length of state-owned large and medium-sized coal mines each year new drivage roadway up to $8000 \mathrm{~km}$ or so, to keep roadway and surrounding rock stability of coal mine construction and production safety is of great significance.

Bolt support in coal mines has become a main supporting way,in coal mine roadway, as a safe and efficient manner, it profoundly changed mine exploiting deployment and roadway arrangement, to high yield and efficient mine construction in China, coal production and benefit of increased significantly and the improvement of the security situation, it plays an irreplaceable role. Anchor or Bolt structure is shown in Fig.1.

Malan mine in gujiao mining area is a new type of modern mine,it is the national state-owned key coal mines during the construction coal mines, which belong to shanxi xishan coal electricity group company. Malan mine construction began in November 1983, in June 1990 formally put into production, the design annual production capacity of 4 million tons. With the mine coal preparation plant in October 1993 and put into production.Malan mine field area of 104.4 square kilometers, 2005 survey by the end of recoverable reserves of 812 million tons, fair service in 145.years.Main products 
are after washing and processing of fertilizer plant, the products are sold to more than 30 domestic steel mills, and exported to Japan, South Korea, India and other international markets. Malan coal mine is shown in Fig.2. Anchor or Bolt truncation processing is shown in Fig.3.

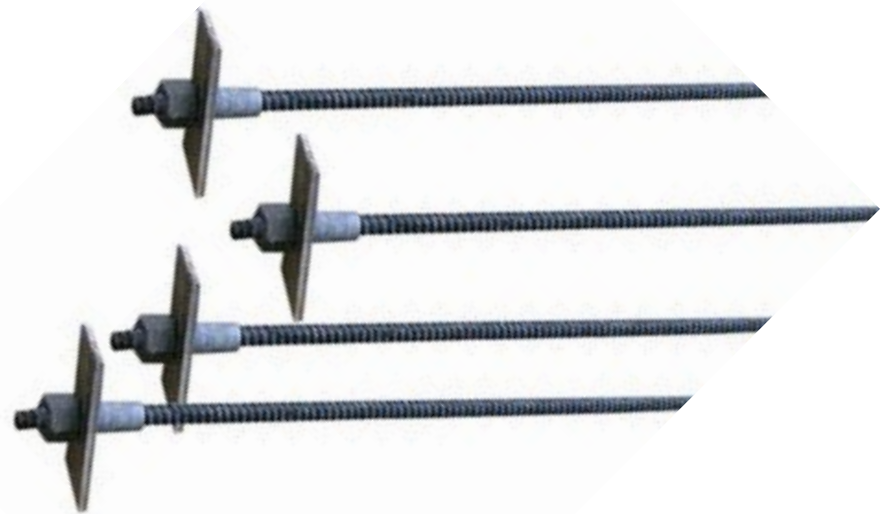

Fig.1 Anchor or Bolt structure

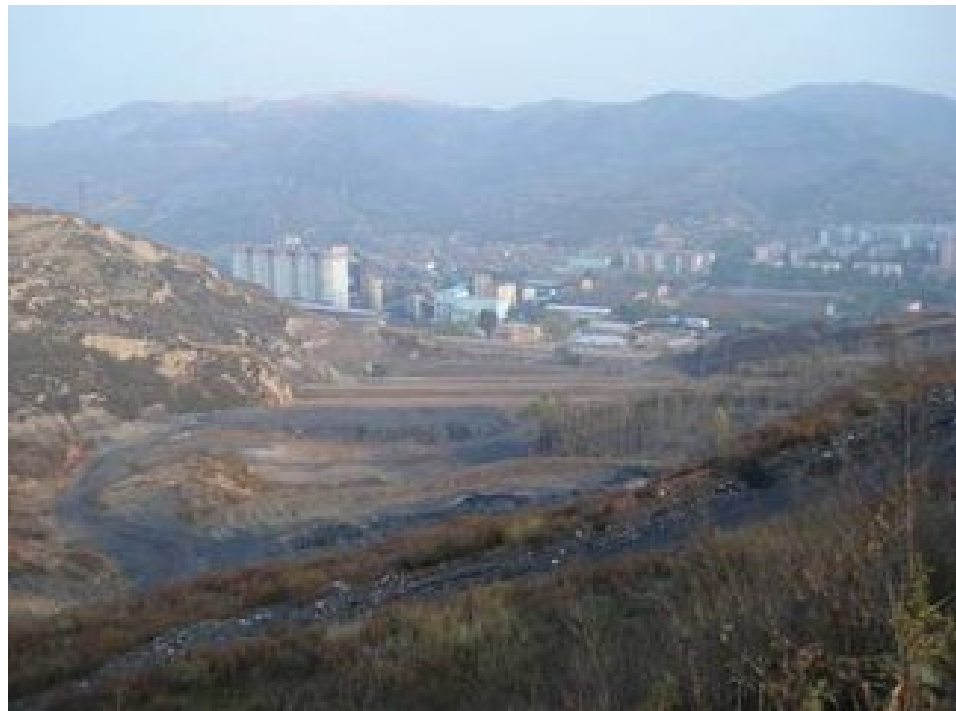

Fig.2 Malan coal mine 


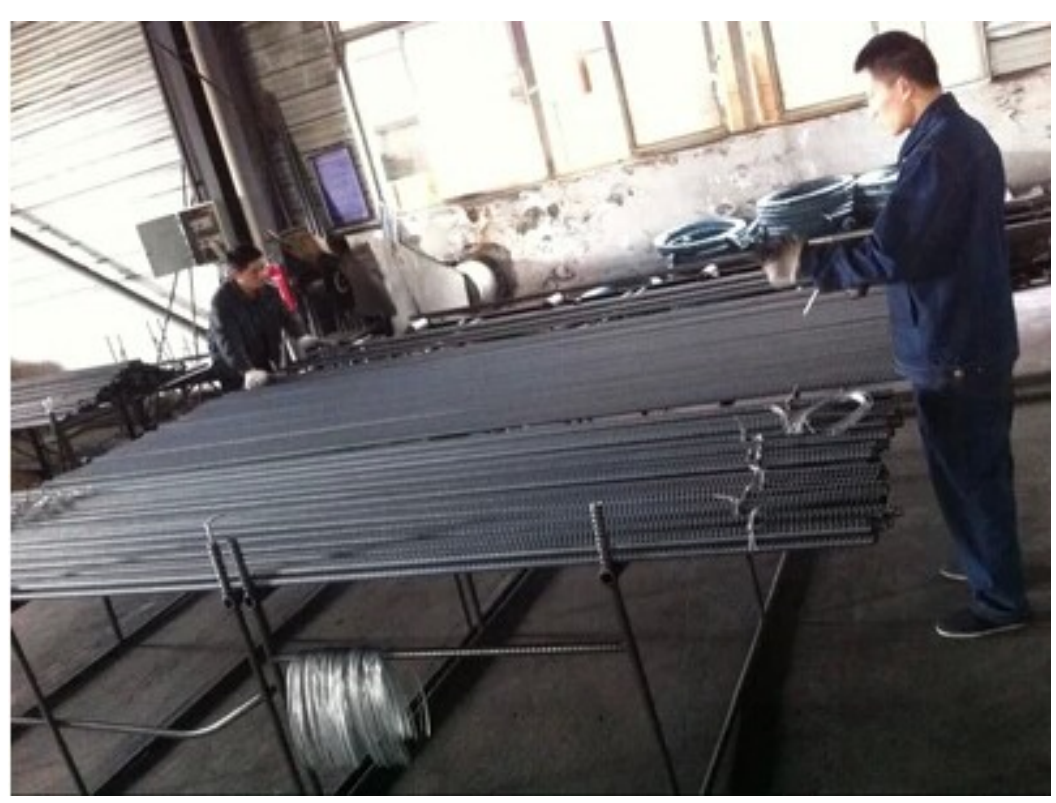

Fig.3 Anchor or Bolt truncation processing

\section{Mathematical Model of Problem}

In mining engineering excavation supporting, A lot of anchor or Bolt is often used in roadway support engineering. due to different geological conditions while supporting, the anchor length is also different. For a given length of the steel bar, how to choose truncation scheme, so that minimizing of the steel bar materials is consumed ?

This problems is often encountered in the actual engineering.

Ttransport gateway is excavated in Malan coal mine, the three kinds of bolt or anchor are need, the length of three kinds of bolt or anchor are respectively $1.5 \mathrm{~m}, 2 \mathrm{~m}, 2.6 \mathrm{~m}$, requirement of these three kinds of bolt or anchor are respectively 7000,1000. 500, But Every materia steel bar length is 5m, How to select the optimal Anchor truncation scheme, and how to select the bolt cutting scheme, so that it makes the consumed steel bar materials at least?

According to the actual problem, we set up the following mathematical model is shown in Table 1 
Table 1 Anchor truncation scheme

\begin{tabular}{|c|c|c|c|c|}
\hline \multirow[b]{2}{*}{ Anchor truncation scheme } & \multicolumn{3}{|c|}{ Anchor length ( m) } & \multirow[b]{2}{*}{ The rest of material } \\
\hline & $\begin{array}{r}\mathrm{L}=1.5 \mathrm{~m} \\
\text { Anch }\end{array}$ & $\begin{array}{l}\mathrm{L}=2.0 \mathrm{~m} \\
\text { Itting root }\end{array}$ & $\begin{array}{l}\mathrm{L}=2.6 \mathrm{~m} \\
\text { nber }\end{array}$ & \\
\hline $\mathrm{I}$ & 2 & 1 & 0 & 0 \\
\hline II & 3 & 0 & 0 & 0.5 \\
\hline III & 0 & 1 & 1 & 0.4 \\
\hline IV & 1 & 0 & 1 & 0.9 \\
\hline $\mathrm{V}$ & 0 & 2 & 0 & 1.0 \\
\hline Sum & 7000 & 1000 & 500 & \\
\hline
\end{tabular}

\section{Solution of the Mathematical Model}

In Table 1, We assume that the bolts are cut $x_{1}$ with Anchor truncation scheme I , the bolts are cut $x_{2}$ with Anchor truncation scheme II , the bolts are cut $x_{3}$ with Anchor truncation scheme III, the bolts are cut $x_{4}$ with Anchor truncation scheme IV, the bolts are cut $x_{5}$ with Anchor truncation scheme $\mathrm{V}$, The above question Can be established the following molding of linear programming:

$$
\begin{gathered}
\text { MinZ }=x_{1}+x_{2}+x_{3}+x_{4}+x_{5} \\
2 x_{1}+3 x_{2}+x_{4}=7000 \\
x_{1}+x_{3}+2 x_{5}=1000 \\
x_{3}+x_{4}=500 \\
x_{1}, x_{2}, x_{3}, x_{4}, x_{5} \geq 0
\end{gathered}
$$


Where $\mathrm{Z}$ is steel raw materials consumed for the total number of root. For formula (1) the linear programming model, available method of simplex tableau.The calculation results as follows:

$$
\begin{aligned}
& \text { MinZ }=3000 \\
& x_{1} *=500 \\
& x_{2} *=2000 \\
& x_{3} *=500 \\
& x_{4} *=0 \\
& x_{5} *=0
\end{aligned}
$$

\section{The Significance of the Calculation Results}

For formula (2), the significance of the calculation results, the optimal solution, the bolts are cut 500 with Anchor truncation scheme I, the bolts are cut 2000, with Anchor truncation scheme II , the bolts are cut 500 with Anchor truncation scheme III , the bolts are cut without Anchor truncation scheme IV, the bolts are cut without Anchor truncation scheme $\mathrm{V}$, both 7000 bolt or anchor with the length of $1.5 \mathrm{~m}, 1000$ bolt or anchor with the length of $2.0 \mathrm{~m}, 500$ bolt or anchor with the length of $2.6 \mathrm{~m}$ can be obtained, and that makes the sum of consumed steel bar materials at least, Thus, using system engineering, optimization ideas and methods, to solve the actual problem is very meaningful.

\section{Acknowledgement}

This research is funded by " The Natural science foundation project of liaoning province of China (201602038)” and “The general Project of scientific research of Education office of Liaoning Province of China (L2012436)”

\section{References}

[1] Wen-an zhou, sixth volume of mine roadway engineering, Beijing: coal industry publishing house, 1983.

[2] De Le, etc., Operations research, Beijing: tsinghua university press, 1992.

[3] Yutian Zheng, The elastic-plastic viscous theories of rock mechanics, Beijing: coal industry publishing house, 1988.

[4] C.A.Tang, Numerical simulation of progressive rock failure and associated seismicity[J]. Int J Rock Mech Min Sci, 1997.34(2):249-261

[5] C.A.Tang,. etc., ,Fracture spacing in layered materials and polygonal fracture [J] Physical Review E, 2006, 73(3): 70-83 